\title{
Feasibility of Compressed Chemotherapy Pediatric Ewing Sarcoma Regimen In Pakistan
}

\author{
Haleema Saeed, Rishm Ali and Saadiya Javed Khan \\ Department of Paediatric Oncology, Shaukat Khanum Memorial Cancer Hospital and Research Centre, Lahore, Pakistan
}

\begin{abstract}
A retrospective chart review was conducted to determine the feasibility of interval-compressed chemotherapy regimen in pediatric Ewing sarcoma (ES) patients treated at Department of Pediatric Oncology, Shaukat Khanum Memorial Trust Cancer Hospital, Lahore, Pakistan, from June 2017 to December 2018. Data was collected regarding demographics, treatment duration and timing, complications and outcome. Completion of treatment within 8 months as considered on time. There were 24 patients (male: female ratio $=$ $1.4: 1$, average age $=10.2 \pm 5.3$ years. Involved sites were extremity in $13(54 \%)$, pelvis in $4(16.6 \%)$, and spine in $3(12.5 \%)$ patients. Twenty patients (83.3\%) completed chemotherapy on time. The mean duration of treatment was $7.2 \pm 1.2$ months. Only three patients $(12 \%)$ exceeded 8 months of duration of treatment. There was an average of 2.5 febrile neutropenia events per patient. Seventeen (70.8\%) patients are alive at the time of review. Two patients died of sepsis, one developed cardiac failure, and one died of progressive disease. This single-institution review showed that patients can tolerate interval-compressed chemotherapy protocol regimen for ES with adequate supportive care.
\end{abstract}

Key Words: Ewing sarcoma, chemotherapy, Compressed regimen, feasibility, Pediatrics.

How to cite this article: Saeed H, Ali R, Khan SJ. Feasibility of Compressed Chemotherapy Pediatric Ewing Sarcoma Regimen In Pakistan. J Coll Physicians Surg Pak 2020; 30(04):446-447. DOI: https://doi.org/10.29271/jcpsp.2020.04.446.

Ewing sarcoma ES is the second most common bone tumor in children with overall survival of $70 \%$ for non-metastatic ES in developed countries. ${ }^{1}$ Survival in low-middle-income countries (LMICs) is still inferior. This can be improved with adherence to protocol therapy and improvement of supportive care. ${ }^{2}$

The aim of this study was to determine the feasibility of switching our current EE99 protocol to the Children's Oncology Group (COG AEWS 0031) compressed chemotherapy protocol, which is now the standard of care in North America. ${ }^{3}$ We believe that if we can deliver chemotherapy in 2-weekly cycles without increasing morbidity and mortality, this can be advantageous in LMICs where inpatient bed limitations and logistical issues of seeking treatment far away from home can be addressed with this shorter protocol.

A retrospective review was conducted for patients with a diagnosis of ES who presented to the Department of Pediatric Oncology, Shaukat Khanum Memorial Trust Cancer Hospital, Lahore, Pakistan, from June 2017 to December 2018.

All patients with histologically proven diagnosis of ES who had received treatment with compressed cycle chemotherapy consisting of alternating VDC (Vincristine, Doxorubicin, Cyclophosphamide) and IE (Ifosphamide, Etoposide) during the study period were included. Exclusion criterion included treat-

Correspondence to: Dr. Saadiya Javed Khan, Department of Paediatric Oncology, Shaukat Khanum Memorial Cancer

Hospital and Research Centre, Lahore, Pakistan

E-mail: saadiyakhan@hotmail.com

Received: May 10, 2019; Revised: September 05, 2020;

Accepted: September 05, 2020

DOI: https://doi.org/10.29271/jcpsp.2020.04.446 ment with different treatment regimen and failure to initiate treatment. Medical records of all eligible patients were retrospectively reviewed after seeking approval from the Institutional Review Board (IRB) at our Hospital. Patients were followed till December 2018. Expected duration of treatment was 7 months. After allowing time for local control, completion of therapy within 8 months was considered to be on-time.

Twenty-four patients were seen during June 2017 to December 2018 (Table I). Male to female ratio was 1.4: 1. Mean age was 10.2 years (range 8 months to 16 years). The average tumor volume was $485 \mathrm{~mL}$ (range 5-1978 mL).

Table l: Patientcharacteristics.

\begin{tabular}{|l|l|}
\hline Age & 10.2 years $( \pm 5.3$ years $)$ \\
\hline Gender (M:F) & $1.4: 1$ \\
\hline Stage & \\
Non-metastatic & $87.5 \%(n=21)$ \\
Metastatic & $12.5 \%(n=3)$ \\
\hline Location & \\
Upper limb & $20.8 \%(n=5)$ \\
Lower limb & $33.3 \%(n=8)$ \\
Pelvis & $16.7 \%(n=4)$ \\
Spine & $12.5 \%(n=3)$ \\
Chest & $8.3 \%(n=2)$ \\
Face & $8.3 \%(n=2)$ \\
\hline
\end{tabular}

All patients received treatment with alternating cycles of VDC and IE every 15 days.

G-CSF was started 24 hours after last dose of chemotherapy and was continued till ANC was above 1000/ $\mu \mathrm{L}$ after nadir. Of the 24 patients, $87 \%(n=21)$ were able to complete therapy on time (Figure 1). Mean duration of treatment was 7.2 \pm 1.2 months. Only three patients (12\%) exceeded 8 months for duration of treat- 
ment. $45 \%(n=11)$ of patients received radiation for local control. Thirteen patients were offered surgical options, $69 \%(n=9)$ had limb salvage surgery, while amputations were done in $31 \%(n=4)$. Timing of local control was variable with only $21 \%(n=5)$ of patients receiving local control after cycle 6 as per the protocol recommendations. However, $54 \%(n=13)$ patients received local control between cycle 6 to 8 . Only $20.8 \%(n=5)$ of patients required admissions for febrile neutropenia. The mean number of admissions for febrile neutropenia was $2.5 \pm 2$.2 episodes.

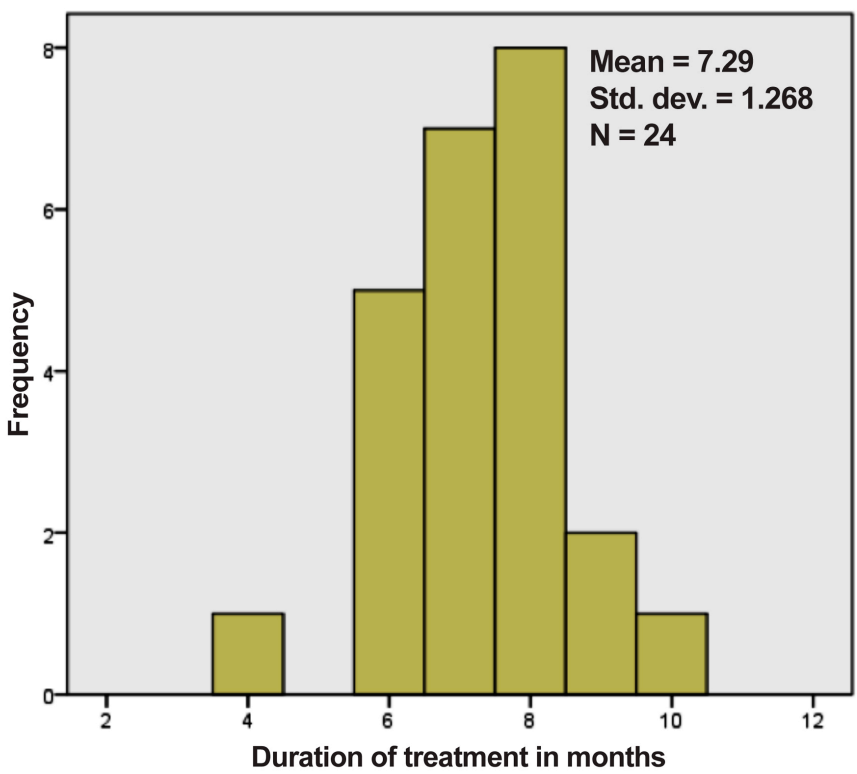

Figure 1: Frequency of duration of treatment of patients.

For the 13 patients who underwent surgical resection $99-100 \%$, necrosis was found in $69 \%(n=9)$ of cases. Of the total, $75 \%(n=18)$ achieved remission. Two out of the three patients $(66.7 \%)$ with metastatic disease failed to achieve remission compared to 4 out of the 21 (19\%) patients with non-metastatic disease with a likelihood ratio of 2.7 for patients with metastatic disease failing to achieveremission.

With a median follow-up time of 11 months (SD 3.7 months), 75\% of the total patients have survived for more than a year. There were four mortalities. Two patients died of sepsis, one developed cardiac failure and one died of progressive disease.

The children's oncology group (COG) has demonstrated that interval compression of chemotherapy from every 21 to 15 days results in improved event-free survival (EFS) from $65 \%$ to $73 \% .{ }^{4}$ The authors were able to demonstrate that interval compression therapy can be achieved in resource limited settings as well. Interval compression therapy was delivered in $88 \%$ patients. Jda et al. demonstrated similar feasibility in Nepal, another developing country. ${ }^{5}$ Majority of the patients completed therapy within 8 months as compared to the patients treated on EuroEwing 99 (EE99) protocol where the planned duration of therapy is 11-12 months. ${ }^{6}$ Many patients in our institution travel from very distant areas and face hardship due to distance from home. The reduction in duration of therapy is likely to increase compliance with comple- tion of therapy. A second advantage of this approach is fewer days of hospitalisation ( 42 days as compared to 54 days). This reduces cost as well as prevents unnecessary delays in chemotherapy due to lack of inpatient beds. There was an excellent initial response to treatment with $70 \%$ of patients showing $99-100 \%$ necrosis on histopathology. Overall $75 \%$ of patients achieved remission.

Our study is limited by short duration of long-term follow-up. However, overall survival of $75 \%$ at 15 months is comparable to that reported in the literature.

Thus, it is feasible to deliver interval-compressed cycle chemotherapy with GCSF support in limited resource settings. This leads to decrease in overall duration of treatment as well as days of hospitalisation. Further long-term follow-up of this cohort is needed to assess continuing survival and complications. Only centers with adequate support should be administering this chemotherapy. In the clinical trial, the observed toxicity was not very different between the standard of care arm ( 3 weeks vs. 2 weeks). Despite that, in general, our population with issues of malnutrition is at risk of increased toxicity especially cardiac toxicity. It is essential to monitor these patients closely for these complications.

\section{CONFLICT OF INTEREST:}

Authors declared no conflict of interest.

\section{AUTHORS' CONTRIBUTION:}

RA: Contributed to data collection and processing. HS, SJK: Equally contributed to results and discussions.

\section{REFERENCES}

1. Esiashvili N, Goodman M, Marcus RB. Changes in incidence and survival of ewing sarcoma patients over the past 3 decades surveillance epidemiology and end results data. J Pediatr Hematol Oncol 2008; 30:425-30.

2. Friedrich P, Ortiz R, Fuentes S, Gamboa Y, Ah Chu-Sanchez MS, Arambú IC, et al. Barriers to effective treatment of pediatric solid tumors in middle-income countries can we make sense of the spectrum of nonbiologic factors that influence outcomes. Cancer 2014; 120:112-25.

3. Reed DR, Hayashi M, Wagner L, Binitie O, Steppan DA, Brohl AS, et al. Treatment pathway of bone sarcoma in children adolescents and young adults. Cancer 2017; 123:2206-18.

4. Womer RB, West DC, Krailo MD, Dickman PS, Pawel BR, Grier $\mathrm{HE}$, et al. Randomized controlled trial of interval-compressed chemotherapy for the treatment of localized Ewing sarcoma a report from the childrens'oncology group. J Clin Oncol 2012; 30:4148-54.

5. Jha AK, Neupane P, Pradhan M, Sharma KS, Shrestha S, Sigdel $P R$, et al. Ewing sarcoma in nepal treated with combined chemotherapy and definitive radiotherapy. J Glob Oncol 2019; 5:1-10.

6. Le Deley M-C, Paulussen M, Lewis I, Brennan B, Ranft A, Whelan J, et al. Cyclophosphamide compared with ifosfamide in consolidation treatment of standard-risk ewing sarcoma results of the randomized noninferiority euro-ewing99-r1 trial. J Clin Oncol 2014; 32:2440-8. 